\title{
Pata contractions and coupled type fixed points
}

\author{
Madjid Eshaghi', Samad Mohseni', Mohsen Rostamian Delavar², Manuel De La Sen³, \\ Gwang Hui Kim ${ }^{4 *}$ and Asma Arian ${ }^{1}$
}

\section{"Correspondence:}

ghkim@kangnam.ac.kr

${ }^{4}$ Department of Mathematics,

Kangnam University, Kangnam,

Korea

Full list of author information is

available at the end of the article

\begin{abstract}
A new coupled fixed point theorem related to the Pata contraction for mappings having the mixed monotone property in partially ordered complete metric spaces is established. It is shown that the coupled fixed point can be unique under some extra suitable conditions involving mid point lower or upper bound properties. Also the corresponding convergence rate is estimated when the iterates of our function converge to its coupled fixed point.
\end{abstract}

MSC: $47 \mathrm{H} 10 ; 34 \mathrm{~B} 15 ; 54 \mathrm{H} 25$

Keywords: partial ordered metric space; mixed monotone property; coupled fixed point

\section{Introduction and preliminaries}

The well-known Banach contraction principle, which guarantees the existence of a unique fixed point for a mapping defined on a complete metric space satisfying the contraction condition, was introduced in 1922 by Banach [1]. After this a great deal of effort has gone into the theory and application of the Banach contraction theorem. Some authors generalized this theorem from the single-valued case to the multivalued [2-4]. Some extensions are to fixed point theorems for contraction mappings in generalized form of metric spaces, especially a metric space endowed with a partial order. Single, coupled, tripled and other types of fixed point theorems are investigated in many works, for instance, [5-9] and references cited therein. Such fixed point theorems are applied to establishing the existence of a unique solution to periodic boundary value problems, matrix equations, ordinary differential equations, and integral equations [10-13]. Recently Pata in [14] introduced a fixed point theorem with weaker hypotheses than those of the Banach contraction principle with an explicit estimate of the convergence rate (see also [15]). Motivated by [16], we establish a new coupled fixed point theorem related to the Pata contraction for mappings having the mixed monotone property in partially ordered metric spaces. We prove that the coupled fixed point can be unique under some suitable conditions. Also the corresponding convergence rate is estimated when the iterates of our function converge to its coupled fixed point.

Definition 1.1 [16] Let $(X, \leq)$ be a partially ordered set. The product space $X \times X$ can be endowed with a partial order such that for $(x, y),(u, v) \in X \times X$ we can define $(u, v) \leq$ $(x, y) \Leftrightarrow x \geq u, y \leq v$.

(02014 Eshaghi et al.; licensee Springer. This is an Open Access article distributed under the terms of the Creative Commons Attribution License (http://creativecommons.org/licenses/by/2.0), which permits unrestricted use, distribution, and reproduction in any medium, provided the original work is properly cited. 
Definition 1.2 [16] Let $(X, \leq)$ be a partially ordered set and $F: X \times X \rightarrow X$. We say that $F$ has the mixed monotone property on $X$ if $F(x, y)$ is monotone non-decreasing in $x$ and is monotone non-increasing in $y$, that is, for any $x, y \in X$,

$$
\begin{array}{lll}
x_{1}, x_{2} \in X, & x_{1} \leq x_{2} \quad \Rightarrow \quad F\left(x_{1}, y\right) \leq F\left(x_{2}, y\right) \quad \text { and } \\
y_{1}, y_{2} \in X, & y_{1} \leq y_{2} \quad \Rightarrow \quad F\left(x, y_{1}\right) \geq F\left(x, y_{2}\right) .
\end{array}
$$

Definition 1.3 [16] An element $(x, y) \in X \times X$ is called a coupled fixed point of the mapping $F: X \times X \rightarrow X$ if

$$
x=F(x, y), \quad y=F(y, x) .
$$

\section{Main results}

In this section we prove a coupled type fixed point theorem with the convergence rate estimation. Also an example is given as an application of the main theorem.

Definition 2.1 Let $(X, d)$ be a metric space. The mapping $\bar{d}: X^{2} \times X^{2} \rightarrow[0, \infty)$ given by

$$
\bar{d}[(x, y),(u, v)]=d(x, u)+d(y, v),
$$

for each pair $((x, y),(u, v)) \in X^{2} \times X^{2}$, defines a metric on $X^{2} \times X^{2}$, which will be denoted for convenience by $d$, too.

For a metric space $(X, d)$, selecting an arbitrary $\left(x_{0}, y_{0}\right) \in X \times X$, we denote

$$
\|x, y\|=d\left[(x, y),\left(x_{0}, y_{0}\right)\right] \quad \text { for all }(x, y) \in X \times X
$$

Let $\psi:[0,1] \rightarrow[0, \infty)$ be an increasing function vanishing with continuity at zero. Also consider the vanishing sequence depending on $\alpha \geq 1, w_{n}(\alpha)=\left(\frac{\alpha}{n}\right)^{\alpha} \sum_{k=1}^{n} \psi\left(\frac{\alpha}{k}\right)$.

Theorem 2.2 Let $(X, \leq)$ be a partially ordered set and suppose that there is a metric $d$ on $X$ such that $(X, d)$ is a complete metric space. Let $F: X \times X \rightarrow X$ be a continuous mapping having the mixed monotone property on $X$ and let $\Lambda \geq 0, \alpha \geq 1$, and $\beta \in[0, \alpha]$ be fixed constants. Suppose that there exist $x_{0}, y_{0} \in X$ such that

$$
x_{0} \leq F\left(x_{0}, y_{0}\right) \text { and } y_{0} \geq F\left(y_{0}, x_{0}\right) \text {. }
$$

If the inequality

$$
d(F(x, y), F(u, v)) \leq \frac{(1-\varepsilon)}{2} d[(x, y),(u, v)]+\Lambda \varepsilon^{\alpha} \psi(\varepsilon)[1+\|x, y\|+\|u, v\|]^{\beta}
$$

is satisfied for every $\varepsilon \in[0,1]$ and $(x, y),(u, v) \in X \times X$ with $u \leq x, y \leq v$, then $F$ has a coupled fixed point $\left(x^{*}, y^{*}\right)$.

Furthermore, we denote $F^{n}=F \circ \cdots \circ F$ ( $n$ times $)$,

$$
d\left[\left(x^{*}, y^{*}\right),\left(F^{n}\left(x_{0}, y_{0}\right), F^{n}\left(y_{0}, x_{0}\right)\right)\right] \leq K w_{n}(\alpha)
$$

for some positive constant $K \leq 2 \Lambda\left(1+4\left\|x^{*}, y^{*}\right\|+4 d\left(x_{0}, y_{0}\right)\right)^{\beta}$. 
Proof Set $F\left(x_{0}, y_{0}\right)=x_{1}$ and $F\left(y_{0}, x_{0}\right)=y_{1}$. So

$$
x_{1} \geq x_{0}, \quad y_{1} \leq y_{0}
$$

Now from letting $x_{2}=F\left(x_{1}, y_{1}\right), y_{2}=F\left(y_{1}, x_{1}\right)$ and choosing the notations

$$
\begin{aligned}
& F^{2}\left(x_{0}, y_{0}\right)=F\left(F\left(x_{0}, y_{0}\right), F\left(y_{0}, x_{0}\right)\right)=F\left(x_{1}, y_{1}\right)=x_{2}, \\
& F^{2}\left(y_{0}, x_{0}\right)=F\left(F\left(y_{0}, x_{0}\right), F\left(x_{0}, y_{0}\right)\right)=F\left(y_{1}, x_{1}\right)=y_{2},
\end{aligned}
$$

with the mixed monotone property of $F$ we have

$$
\begin{aligned}
& x_{2}=F^{2}\left(x_{0}, y_{0}\right)=F\left(x_{1}, y_{1}\right) \geq F\left(x_{0}, y_{0}\right)=x_{1}, \\
& y_{2}=F^{2}\left(y_{0}, x_{0}\right)=F\left(y_{1}, x_{1}\right) \leq F\left(y_{0}, x_{0}\right)=y_{1} .
\end{aligned}
$$

So by continuing this argument we obtain two sequences $\left\{x_{n}\right\}_{n=0}^{\infty}$ and $\left\{y_{n}\right\}_{n=0}^{\infty}$ such that $x_{0} \leq x_{1} \leq \cdots \leq x_{n} \leq \cdots$ and $y_{0} \geq y_{1} \geq \cdots \geq y_{n} \geq \cdots$.

Furthermore for $n=1,2, \ldots$ we set

$$
\begin{aligned}
& x_{n}=F\left(x_{n-1}, y_{n-1}\right)=F^{n}\left(x_{0}, y_{0}\right), \quad y_{n}=F\left(y_{n-1}, x_{n-1}\right)=F^{n}\left(y_{0}, x_{0}\right) \quad \text { and } \\
& C_{n}=\left\|F^{n}\left(x_{0}, y_{0}\right), F^{n}\left(y_{0}, x_{0}\right)\right\| .
\end{aligned}
$$

Since (2.1) is true for every $\varepsilon \in[0,1]$, setting $\varepsilon=0$ we have the following relations:

$$
\begin{aligned}
& d\left[\left(F^{n+1}\left(x_{0}, y_{0}\right), F^{n+1}\left(y_{0}, x_{0}\right)\right),\left(F^{n}\left(x_{0}, y_{0}\right), F^{n}\left(y_{0}, x_{0}\right)\right)\right] \leq C_{1}, \\
& d\left[\left(F^{n+1}\left(x_{0}, y_{0}\right), F^{n+1}\left(y_{0}, x_{0}\right)\right),\left(x_{0}, y_{0}\right)\right] \\
& \quad \leq d\left[\left(F^{n+1}\left(x_{0}, y_{0}\right), F^{n+1}\left(y_{0}, x_{0}\right)\right),\left(F\left(x_{0}, y_{0}\right), F\left(y_{0}, x_{0}\right)\right)\right] \\
& \quad+d\left[\left(F\left(x_{0}, y_{0}\right), F\left(y_{0}, x_{0}\right)\right),\left(x_{0}, y_{0}\right)\right], \\
& d\left[\left(F^{n}\left(x_{0}, y_{0}\right), F^{n}\left(y_{0}, x_{0}\right)\right),\left(x_{0}, y_{0}\right)\right] \\
& \quad \leq d\left[\left(F^{n}\left(x_{0}, y_{0}\right), F^{n}\left(y_{0}, x_{0}\right)\right),\left(F^{n+1}\left(x_{0}, y_{0}\right), F^{n+1}\left(y_{0}, x_{0}\right)\right)\right] \\
& \quad+d\left[\left(F^{n+1}\left(x_{0}, y_{0}\right), F^{n+1}\left(y_{0}, x_{0}\right)\right),\left(x_{0}, y_{0}\right)\right] .
\end{aligned}
$$

Using (2.5), (2.3), (2.4), and (2.1) we have

$$
\begin{aligned}
C_{n} \leq & d\left[\left(F^{n+1}\left(x_{0}, y_{0}\right), F^{n+1}\left(y_{0}, x_{0}\right)\right),\left(F\left(x_{0}, y_{0}\right), F\left(y_{0}, x_{0}\right)\right)\right]+2 C_{1} \\
= & d\left(F^{n+1}\left(x_{0}, y_{0}\right), F\left(x_{0}, y_{0}\right)\right)+d\left(F^{n+1}\left(y_{0}, x_{0}\right), F\left(y_{0}, x_{0}\right)\right)+2 C_{1} \\
= & d\left[F\left(F^{n}\left(x_{0}, y_{0}\right), F^{n}\left(y_{0}, x_{0}\right)\right), F\left(x_{0}, y_{0}\right)\right] \\
& +d\left[F\left(F^{n}\left(y_{0}, x_{0}\right), F^{n}\left(x_{0}, y_{0}\right)\right), F\left(y_{0}, x_{0}\right)\right]+2 C_{1} \\
\leq & \frac{(1-\varepsilon)}{2} d\left[\left(F^{n}\left(x_{0}, y_{0}\right), F^{n}\left(y_{0}, x_{0}\right)\right),\left(x_{0}, y_{0}\right)\right] \\
& +\Lambda \varepsilon^{\alpha} \psi(\varepsilon)\left[1+\left\|F^{n}\left(x_{0}, y_{0}\right), F^{n}\left(y_{0}, x_{0}\right)\right\|+\left\|x_{0}, y_{0}\right\|\right]^{\beta}
\end{aligned}
$$




$$
\begin{aligned}
& +\frac{(1-\varepsilon)}{2} d\left[\left(F^{n}\left(y_{0}, x_{0}\right), F^{n}\left(x_{0}, y_{0}\right)\right),\left(y_{0}, x_{0}\right)\right] \\
& +\Lambda \varepsilon^{\alpha} \psi(\varepsilon)\left[1+\left\|F^{n}\left(y_{0}, x_{0}\right), F^{n}\left(x_{0}, y_{0}\right)\right\|+\left\|y_{0}, x_{0}\right\|\right]^{\beta} \\
& +2 C_{1} .
\end{aligned}
$$

But from the definition of $\|\cdot\|$ we have

$$
\begin{aligned}
& {\left[1+\left\|F^{n}\left(x_{0}, y_{0}\right), F^{n}\left(y_{0}, x_{0}\right)\right\|+\left\|x_{0}, y_{0}\right\|\right] \leq 1+C_{n} \leq 1+C_{n}+4 d\left(x_{0}, y_{0}\right),} \\
& {\left[1+\left\|F^{n}\left(y_{0}, x_{0}\right), F^{n}\left(x_{0}, y_{0}\right)\right\|+\left\|y_{0}, x_{0}\right\|\right] \leq 1+C_{n}+4 d\left(x_{0}, y_{0}\right),}
\end{aligned}
$$

so for $\alpha \geq \beta$ there are some $E, D>0$ such that

$$
\begin{aligned}
C_{n} \leq & \frac{(1-\varepsilon)}{2} C_{n}+\Lambda \varepsilon^{\alpha} \psi(\varepsilon)\left[1+C_{n}+4 d\left(x_{0}, y_{0}\right)\right]^{\beta} \\
& +\frac{(1-\varepsilon)}{2} C_{n}+\Lambda \varepsilon^{\alpha} \psi(\varepsilon)\left[1+C_{n}+4 d\left(x_{0}, y_{0}\right)\right]^{\beta}+2 C_{1} \\
= & (1-\varepsilon) C_{n}+2 \Lambda \varepsilon^{\alpha} \psi(\varepsilon)\left[1+C_{n}+4 d\left(x_{0}, y_{0}\right)\right]^{\beta}+2 C_{1} \leq(1-\varepsilon) C_{n}+E \varepsilon^{\alpha} \psi(\varepsilon) C_{n}^{\alpha}+D .
\end{aligned}
$$

Accordingly,

$$
\varepsilon C_{n} \leq E \varepsilon^{\alpha} \psi(\varepsilon) C_{n}^{\alpha}+D
$$

which holds by hypothesis for any $\varepsilon \in[0,1]$ taken for each $n \in \mathbb{N}$. If there is a subsequence $C_{n_{k}} \rightarrow \infty$, then the choice $\varepsilon_{n_{k}}=\min \left(1, \frac{1+D}{C_{n_{k}}}\right)$ leads to the following contradiction:

$$
1 \leq E(1+D)^{\alpha} \psi\left(\varepsilon_{n_{k}}\right) \rightarrow 0 \quad \text { as } n_{k} \rightarrow \infty
$$

Then the sequence $\left\{C_{n}\right\}_{n=1}^{\infty}$ is bounded. Also the sequences $\left\{F^{n}\left(x_{0}, y_{0}\right)\right\}$ and $\left\{F^{n}\left(y_{0}, x_{0}\right)\right\}$ are Cauchy sequences:

$$
\begin{aligned}
& d\left(F^{n+m+1}\left(x_{0}, y_{0}\right), F^{n+1}\left(x_{0}, y_{0}\right)\right)+d\left(F^{n+m+1}\left(y_{0}, x_{0}\right), F^{n+1}\left(y_{0}, x_{0}\right)\right) \\
&=d\left(F\left(F^{n+m}\left(x_{0}, y_{0}\right), F^{n+m}\left(y_{0}, x_{0}\right)\right), F\left(F^{n}\left(x_{0}, y_{0}\right), F^{n}\left(y_{0}, x_{0}\right)\right)\right) \\
& \quad+d\left(F\left(F^{n+m}\left(y_{0}, x_{0}\right), F^{n+m}\left(x_{0}, y_{0}\right)\right), F\left(F^{n}\left(y_{0}, x_{0}\right), F^{n}\left(x_{0}, y_{0}\right)\right)\right) \\
& \leq \frac{(1-\varepsilon)}{2} d\left[\left(F^{n+m}\left(x_{0}, y_{0}\right), F^{n+m}\left(y_{0}, x_{0}\right)\right),\left(F^{n}\left(x_{0}, y_{0}\right), F^{n}\left(y_{0}, x_{0}\right)\right)\right] \\
&+\Lambda \varepsilon^{\alpha} \psi(\varepsilon)\left[1+\left\|F^{n+m}\left(x_{0}, y_{0}\right), F^{n+m}\left(y_{0}, x_{0}\right)\right\|+\left\|F^{n}\left(x_{0}, y_{0}\right), F^{n}\left(y_{0}, x_{0}\right)\right\|\right]^{\beta} \\
&+\frac{(1-\varepsilon)}{2} d\left[\left(F^{n+m}\left(y_{0}, x_{0}\right), F^{n+m}\left(x_{0}, y_{0}\right)\right),\left(F^{n}\left(y_{0}, x_{0}\right), F^{n}\left(x_{0}, y_{0}\right)\right)\right] \\
&+\Lambda \varepsilon^{\alpha} \psi(\varepsilon)\left[1+\left\|F^{n+m}\left(y_{0}, x_{0}\right), F^{n+m}\left(x_{0}, y_{0}\right)\right\|+\left\|F^{n}\left(y_{0}, x_{0}\right), F^{n}\left(x_{0}, y_{0}\right)\right\|\right]^{\beta} .
\end{aligned}
$$

But since

$$
\begin{aligned}
& \left\|F^{n+m}\left(x_{0}, y_{0}\right), F^{n+m}\left(y_{0}, x_{0}\right)\right\|+\left\|F^{n}\left(x_{0}, y_{0}\right), F^{n}\left(y_{0}, x_{0}\right)\right\| \\
& \quad \leq c_{n+m}+c_{n} \leq c_{n+m}+c_{n}+4 d\left(x_{0}, y_{0}\right)
\end{aligned}
$$




$$
\left\|F^{n+m}\left(y_{0}, x_{0}\right), F^{n+m}\left(x_{0}, y_{0}\right)\right\|+\left\|F^{n}\left(y_{0}, x_{0}\right), F^{n}\left(x_{0}, y_{0}\right)\right\| \leq c_{n+m}+c_{n}+4 d\left(x_{0}, y_{0}\right)
$$

we have

$$
\begin{aligned}
d( & \left.F^{n+m+1}\left(x_{0}, y_{0}\right), F^{n+1}\left(x_{0}, y_{0}\right)\right)+d\left(F^{n+m+1}\left(y_{0}, x_{0}\right), F^{n+1}\left(y_{0}, x_{0}\right)\right) \\
\leq & (1-\varepsilon)\left(d\left(F^{n+m}\left(x_{0}, y_{0}\right), F^{n}\left(x_{0}, y_{0}\right)\right)+d\left(F^{n+m}\left(y_{0}, x_{0}\right), F^{n}\left(y_{0}, x_{0}\right)\right)\right) \\
& +2 \Lambda \varepsilon^{\alpha} \psi(\varepsilon)\left[1+c_{n+m}+c_{n}+4 d\left(x_{0}, y_{0}\right)\right]^{\beta} .
\end{aligned}
$$

For fixed $m$, set

$$
K=\sup _{n \in N} 2 \Lambda\left[1+2 c_{n}+4 d\left(x_{0}, y_{0}\right)\right]^{\beta}
$$

and $\varepsilon=1-\left(\frac{n}{n+1}\right)^{\alpha} \leq \frac{\alpha}{n+1}$. So

$$
\begin{aligned}
& (n+1)^{\alpha}\left(d\left(F^{n+m+1}\left(x_{0}, y_{0}\right), F^{n+1}\left(x_{0}, y_{0}\right)\right)+d\left(F^{n+m+1}\left(y_{0}, x_{0}\right), F^{n+1}\left(y_{0}, x_{0}\right)\right)\right) \\
& \quad \leq n^{\alpha}\left(d\left(F^{n+m}\left(x_{0}, y_{0}\right), F^{n}\left(x_{0}, y_{0}\right)\right)+d\left(F^{n+m}\left(y_{0}, x_{0}\right), F^{n}\left(y_{0}, x_{0}\right)\right)\right)+K \alpha^{\alpha} \psi\left(\frac{\alpha}{n+1}\right) .
\end{aligned}
$$

Setting $r_{n}:=n^{\alpha}\left[d\left(F^{n+m}\left(x_{0}, y_{0}\right), F^{n}\left(x_{0}, y_{0}\right)\right)+d\left(F^{n+m}\left(y_{0}, x_{0}\right), F^{n}\left(y_{0}, x_{0}\right)\right)\right]$ we have

$$
\begin{aligned}
r_{n+1} & \leq r_{n}+K \alpha^{\alpha} \psi\left(\frac{\alpha}{n+1}\right) \\
& \leq r_{n-1}+K \alpha^{\alpha} \psi\left(\frac{\alpha}{n}\right)+K \alpha^{\alpha} \psi\left(\frac{\alpha}{n+1}\right) \\
& \leq \cdots \\
& \leq r_{0}+K \alpha^{\alpha} \sum_{k=1}^{n+1} \psi\left(\frac{\alpha}{k}\right)=K \alpha^{\alpha} \sum_{k=1}^{n+1} \psi\left(\frac{\alpha}{k}\right) .
\end{aligned}
$$

Therefore

$$
\begin{aligned}
& d\left(F^{n+m}\left(x_{0}, y_{0}\right), F^{n}\left(x_{0}, y_{0}\right)\right)+d\left(F^{n+m}\left(y_{0}, x_{0}\right), F^{n}\left(y_{0}, x_{0}\right)\right) \\
& \quad \leq K\left(\frac{\alpha}{n}\right)^{\alpha} \sum_{k=1}^{n} \psi\left(\frac{\alpha}{k}\right)=K w_{n}(\alpha) .
\end{aligned}
$$

Taking limits as $n \rightarrow \infty$, we get $d\left(F^{n+m}\left(x_{0}, y_{0}\right), F^{n}\left(x_{0}, y_{0}\right)\right)+d\left(F^{n+m}\left(y_{0}, x_{0}\right), F^{n}\left(y_{0}, x_{0}\right)\right) \rightarrow 0$. This implies that $\left\{F^{n}\left(x_{0}, y_{0}\right)\right\}$ and $\left\{F^{n}\left(y_{0}, x_{0}\right)\right\}$ are Cauchy sequences in $X$. Since $X$ is a complete metric space, there are $x^{*}, y^{*} \in X$ such that

$$
\lim _{n \rightarrow \infty} F^{n}\left(x_{0}, y_{0}\right)=\lim _{n \rightarrow \infty} x_{n}=x^{*} \quad \text { and } \quad \lim _{m \rightarrow \infty} F^{m}\left(y_{0}, x_{0}\right)=\lim _{m \rightarrow \infty} y_{m}=y^{*} .
$$

Using (2.7) and Definition 2.1 we have

$$
\begin{array}{r}
\lim _{n \rightarrow \infty} d\left[\left(x^{*}, y^{*}\right),\left(F\left(x_{n-1}, y_{n-1}\right), F\left(y_{n-1}, x_{n-1}\right)\right)\right] \\
\quad=\lim _{n \rightarrow \infty} d\left[\left(x^{*}, y^{*}\right),\left(F^{n}\left(x_{0}, y_{0}\right), F^{n}\left(y_{0}, x_{0}\right)\right)\right]
\end{array}
$$




$$
\begin{aligned}
& =\lim _{n, m \rightarrow \infty} d\left[\left(F^{n+m}\left(x_{0}, y_{0}\right), F^{n+m}\left(y_{0}, x_{0}\right)\right),\left(F^{n}\left(x_{0}, y_{0}\right), F^{n}\left(y_{0}, x_{0}\right)\right)\right] \\
& \leq K w_{n}(\alpha)
\end{aligned}
$$

and from the continuity of $F$ we have $F\left(x^{*}, y^{*}\right)=x^{*}$ and $F\left(y^{*}, x^{*}\right)=y^{*}$. Also, the convergence rate estimate stated in (2.2) is obtained from the following relations:

$$
\begin{aligned}
d[ & \left.\left(x^{*}, y^{*}\right),\left(F^{n}\left(x_{0}, y_{0}\right), F^{n}\left(y_{0}, x_{0}\right)\right)\right] \\
= & d\left[\left(F\left(x^{*}, y^{*}\right), F\left(y^{*}, x^{*}\right)\right),\left(F^{n}\left(x_{0}, y_{0}\right), F^{n}\left(y_{0}, x_{0}\right)\right)\right] \\
= & d\left(F\left(x^{*}, y^{*}\right), F^{n}\left(x_{0}, y_{0}\right)\right)+d\left(F\left(y^{*}, x^{*}\right), F^{n}\left(y_{0}, x_{0}\right)\right) \\
\leq & 1 / 2 d\left[\left(x^{*}, y^{*}\right),\left(F^{n-1}\left(x_{0}, y_{0}\right), F^{n-1}\left(y_{0}, x_{0}\right)\right)\right] \\
& +1 / 2 d\left[\left(y^{*}, x^{*}\right),\left(F^{n-1}\left(y_{0}, x_{0}\right), F^{n-1}\left(x_{0}, y_{0}\right)\right)\right] \\
= & d\left[\left(x^{*}, y^{*}\right),\left(F^{n-1}\left(x_{0}, y_{0}\right), F^{n-1}\left(y_{0}, x_{0}\right)\right)\right] \\
& \vdots \\
\leq & d\left[\left(x^{*}, y^{*}\right),\left(x_{0}, y_{0}\right)\right]=\left\|x^{*}, y^{*}\right\|,
\end{aligned}
$$

which implies that

$$
\begin{aligned}
c_{n} & =d\left[\left(F^{n}\left(x_{0}, y_{0}\right), F^{n}\left(y_{0}, x_{0}\right)\right),\left(x_{0}, y_{0}\right)\right] \\
& \leq d\left[\left(F^{n}\left(x_{0}, y_{0}\right), F^{n}\left(y_{0}, x_{0}\right)\right),\left(x^{*}, y^{*}\right)\right]+d\left[\left(x^{*}, y^{*}\right),\left(x_{0}, y_{0}\right)\right] \\
& \leq 2\left\|x^{*}, y^{*}\right\| .
\end{aligned}
$$

From the last inequality and (2.6) we have $K \leq 2 \Lambda\left[1+4\left\|x^{*}, y^{*}\right\|+4 d\left(x_{0}, y_{0}\right)\right]^{\beta}$.

Example 2.3 Let $X=[-2,2]$ and $d: X \times X \rightarrow[0, \infty)$ be a metric on $X$ defined as $d(x, y)=$ $|x-y|$ for all $x, y \in X$ which $(X, d)$ is a complete metric space. Now consider $X \times X$ with the partial order defined in Definition 1.1. The metric on the product metric space $X \times X$ is defined by

$$
d\left(\left(x_{1}, y_{1}\right),\left(x_{2}, y_{2}\right)\right)=\left|x_{1}-x_{2}\right|+\left|y_{1}-y_{2}\right|, \quad \text { for all } x_{i}, y_{i} \in X(i=1,2) .
$$

Consider the mapping $F$ defined as

$$
F(x, y)=1,
$$

for all fixed constants $\Lambda \geq 0, \alpha \geq 1$, and $\beta \in[0, \alpha]$, and by using (2.1) we have

$$
0 \leq \frac{(1-\varepsilon)}{2} d[(x, y),(u, v)]+\Lambda \varepsilon^{\alpha} \psi(\varepsilon)[1+\|x, y\|+\|u, v\|]^{\beta} .
$$

Since $d[(x, y),(u, v)]$ and $\Lambda \varepsilon^{\alpha} \psi(\varepsilon)[1+\|x, y\|+\|u, v\|]^{\beta}$ are positive, we have $\frac{(1-\varepsilon)}{2} \geq 0$, which forces $\varepsilon \in[0,1]$. Also there exists $(0,2) \in X \times X$ such that $0 \leq F(0,2)$ and $2 \geq F(2,0)$. Thus $F$ satisfies all conditions of Theorem 2.2. The pair $(1,1)$ is the coupled fixed point for the mapping $F$. 


\section{Supplementary results}

In this section we prove some supplementary results. The first theorem is about replacing the continuity condition of the function $F$ in Theorem 2.2 with a new one. The second is about the uniqueness of the coupled fixed point. At last we show that the hypothesis of Theorem 2.2 is weaker than those of Theorem 2.1 in [16].

Theorem 3.1 Let $(X, \leq)$ be a partially ordered set and suppose that there is a metric $d$ on $X$ such that $(X, d)$ is a complete metric space. Let $F: X \times X \rightarrow X$ be a mapping having the mixed monotone property on $X$ such that there are two elements $x_{0}, y_{0} \in X$ with

$$
x_{0} \leq F\left(x_{0}, y_{0}\right) \quad \text { and } \quad y_{0} \geq F\left(y_{0}, x_{0}\right) .
$$

Let $\Lambda \geq 0, \alpha \geq 1$, and $\beta \in[0, \alpha]$ be fixed constants. Suppose that the inequality

$$
d(F(x, y), F(u, v)) \leq \frac{(1-\varepsilon)}{2} d[(x, y),(u, v)]+\Lambda \varepsilon^{\alpha} \psi(\varepsilon)[1+\|x, y\|+\|u, v\|]^{\beta}
$$

is satisfied for every $\varepsilon \in[0,1]$ and $(x, y),(u, v) \in X \times X$ with $u \leq x, y \leq v$. Furthermore, suppose that $X$ has the following properties:

(i) for a non-decreasing sequence $x_{n} \rightarrow x$, then $x_{n} \leq x$ for all $n \in \mathbb{N}$,

(ii) for a non-increasing sequence $y_{n} \rightarrow y$, then $y_{n} \geq y$ for all $n \in \mathbb{N}$.

Then $F$ has a coupled fixed point $\left(x^{*}, y^{*}\right)$.

Furthermore, calling $F^{n}=F \circ \cdots \circ F$ ( $n$ times), we have

$$
d\left[\left(x^{*}, y^{*}\right),\left(F^{n}\left(x_{0}, y_{0}\right), F^{n}\left(y_{0}, x_{0}\right)\right)\right] \leq K w_{n}(\alpha)
$$

for some positive constant $K \leq 2 \Lambda\left(1+4\left\|x^{*}, y^{*}\right\|+4 d\left(x_{0}, y_{0}\right)\right)^{\beta}$.

Proof We only need to prove that $x^{*}=F\left(x^{*}, y^{*}\right)$ and $y^{*}=F\left(y^{*}, x^{*}\right)$. Consider the following relations:

$$
\begin{aligned}
& d\left[\left(x^{*}, y^{*}\right),\left(F\left(x^{*}, y^{*}\right), F\left(y^{*}, x^{*}\right)\right)\right] \\
& \leq d\left[\left(x^{*}, y^{*}\right),\left(F^{n+1}\left(x_{0}, y_{0}\right), F^{n+1}\left(y_{0}, x_{0}\right)\right)\right] \\
&+d\left[\left(F^{n+1}\left(x_{0}, y_{0}\right), F^{n+1}\left(y_{0}, x_{0}\right)\right),\left(F\left(x^{*}, y^{*}\right), F\left(y^{*}, x^{*}\right)\right)\right] \\
&= d\left[\left(x^{*}, y^{*}\right),\left(F^{n+1}\left(x_{0}, y_{0}\right), F^{n+1}\left(y_{0}, x_{0}\right)\right)\right] \\
&+d\left(F\left(F^{n}\left(x_{0}, y_{0}\right), F^{n}\left(y_{0}, x_{0}\right)\right), F\left(x^{*}, y^{*}\right)\right)+d\left(F\left(F^{n}\left(y_{0}, x_{0}\right), F^{n}\left(x_{0}, y_{0}\right)\right), F\left(y^{*}, x^{*}\right)\right) \\
& \leq d\left[\left(x^{*}, y^{*}\right),\left(F^{n+1}\left(x_{0}, y_{0}\right), F^{n+1}\left(y_{0}, x_{0}\right)\right)\right] \\
&+\frac{(1-\varepsilon)}{2} d\left[\left(F^{n}\left(x_{0}, y_{0}\right), F^{n}\left(y_{0}, x_{0}\right)\right),\left(x^{*}, y^{*}\right)\right] \\
&+\Lambda \varepsilon^{\alpha} \psi(\varepsilon)\left[1+\left\|F^{n}\left(x_{0}, y_{0}\right), F^{n}\left(y_{0}, x_{0}\right)\right\|+\left\|x^{*}, y^{*}\right\|\right]^{\beta} \\
&+\frac{(1-\varepsilon)}{2} d\left[\left(F^{n}\left(y_{0}, x_{0}\right), F^{n}\left(x_{0}, y_{0}\right)\right),\left(y^{*}, x^{*}\right)\right] \\
&+\Lambda \varepsilon^{\alpha} \psi(\varepsilon)\left[1+\left\|F^{n}\left(y_{0}, x_{0}\right), F^{n}\left(x_{0}, y_{0}\right)\right\|+\left\|y^{*}, x^{*}\right\|\right]^{\beta} .
\end{aligned}
$$


Now since

$$
\lim _{n \rightarrow \infty} F^{n}\left(x_{0}, y_{0}\right)=\lim _{n \rightarrow \infty} x_{n}=x^{*} \text { and } \lim _{m \rightarrow \infty} F^{m}\left(y_{0}, x_{0}\right)=\lim _{m \rightarrow \infty} y_{m}=y^{*}
$$

and since the contractive condition (3.1) holds for any real constant $\varepsilon \in[0,1]$, we can replace $\varepsilon$, for each $n \in \mathbb{N}$, by a sequence $[0,1] \ni \varepsilon_{n} \rightarrow 0$ as $n \rightarrow \infty$. Then by letting $\varepsilon=\varepsilon_{n} \rightarrow 0$ as $n \rightarrow \infty$, we have $d\left[\left(x^{*}, y^{*}\right),\left(F\left(x^{*}, y^{*}\right), F\left(y^{*}, x^{*}\right)\right)\right]=0$.

Definition 3.2 Suppose that $X$ is a partially ordered metric space with a metric $d$. A pair of $(x, y),\left(x^{*}, y^{*}\right) \in X \times X$ has either a mid point lower bound or a mid point upper bound if there are $\left(z_{1}, z_{2}\right) \in X \times X$ comparable to $(x, y)$ and $(u, v)$ such that $d\left[(x, y),\left(z_{1}, z_{2}\right)\right]+$ $d\left[\left(z_{1}, z_{2}\right),\left(x^{*}, y^{*}\right)\right]=d\left[(x, y),\left(x^{*}, y^{*}\right)\right]$. The space $(X, d)$ has the mid point lower bound or the mid point upper bound property if any pair in $X \times X$ has a mid point lower bound or a mid point upper bound.

Theorem 3.3 Adding the condition of the above definition to the space $(X, d)$ in the hypothesis of Theorem 3.1, we obtain the uniqueness of the coupled fixed point of $F$.

Proof If $(x, y) \in X \times X$ is another coupled fixed point of $F$ where $x=F(x, y), y=F(y, x)$, then we have two cases.

Case (1): If $\left(x^{*}, y^{*}\right)$ is comparable to $(x, y)$ then

$$
\begin{aligned}
d\left[(x, y),\left(x^{*}, y^{*}\right)\right]= & d\left[(F(x, y), F(y, x)),\left(F\left(x^{*}, y^{*}\right), F\left(y^{*}, x^{*}\right)\right)\right] \\
= & d\left(F(x, y), F\left(x^{*}, y^{*}\right)\right)+d\left(F(y, x), F\left(y^{*}, x^{*}\right)\right) \\
\leq & \frac{(1-\varepsilon)}{2} d\left[(x, y),\left(x^{*}, y^{*}\right)\right]+t_{1} \varepsilon \psi(\varepsilon) \\
& +\frac{(1-\varepsilon)}{2} d\left[(y, x),\left(y^{*}, x^{*}\right)\right]+t_{2} \varepsilon \psi(\varepsilon),
\end{aligned}
$$

for some $t_{1}, t_{2}>0$. Putting $t=t_{1}+t_{2}$, we have

$$
\varepsilon d\left[(x, y),\left(x^{*}, y^{*}\right)\right] \leq \varepsilon t \psi(\varepsilon)
$$

which is valid for every $\varepsilon \in[0,1]$. We can replace $\varepsilon$, for each $n \in \mathbb{N}$, by a sequence $[0,1] \ni$ $\varepsilon_{n} \rightarrow 0$ as $n \rightarrow \infty$ which forces $d\left[(x, y),\left(x^{*}, y^{*}\right)\right]=0$.

Case (2): If $\left(x^{*}, y^{*}\right)$ is not comparable to $(x, y)$ then there exists a mid upper bound or mid lower bound $(u, v) \in X \times X$ for $\left(x^{*}, y^{*}\right)$ and $(x, y)$. So $(F(u, v), F(v, u))$ is comparable to $\left(x^{*}, y^{*}\right)=\left(F\left(x^{*}, y^{*}\right), F\left(y^{*}, x^{*}\right)\right)$ and $(x, y)=(F(x, y), F(y, x))$. It follows that

$$
\begin{aligned}
& d\left[(x, y),\left(x^{*}, y^{*}\right)\right] \\
&= d\left[(F(x, y), F(y, x)),\left(F\left(x^{*}, y^{*}\right), F\left(y^{*}, x^{*}\right)\right)\right] \\
& \leq d[(F(x, y), F(y, x)),(F(u, v), F(v, u))]+d\left[(F(u, v), F(v, u)),\left(F\left(x^{*}, y^{*}\right), F\left(y^{*}, x^{*}\right)\right)\right] \\
&= d(F(x, y), F(u, v))+d(F(y, x), F(v, u))+d\left(F(u, v), F\left(x^{*}, y^{*}\right)\right) \\
&+d\left(F(v, u), F\left(y^{*}, x^{*}\right)\right)
\end{aligned}
$$




$$
\begin{aligned}
\leq & \frac{(1-\varepsilon)}{2} d[(x, y),(u, v)]+t_{1} \varepsilon \psi(\varepsilon)+\frac{(1-\varepsilon)}{2} d[(y, x),(v, u)]+t_{2} \varepsilon \psi(\varepsilon) \\
& +\frac{(1-\varepsilon)}{2} d\left[(u, v),\left(x^{*}, y^{*}\right)\right]+t_{3} \varepsilon \psi(\varepsilon)+\frac{(1-\varepsilon)}{2} d\left[(v, u),\left(y^{*}, x^{*}\right)\right]+t_{4} \varepsilon \psi(\varepsilon),
\end{aligned}
$$

for some $t_{1}, t_{2}, t_{3}, t_{4}>0$. Set $t=t_{1}+t_{2}+t_{3}+t_{4}$. Hence,

$$
d\left[(x, y),\left(x^{*}, y^{*}\right)\right] \leq(1-\varepsilon)\left[d(x, u)+d(y, v)+d\left(u, x^{*}\right)+d\left(v, y^{*}\right)\right]+t \varepsilon \psi(\varepsilon) .
$$

Now by Definition 3.2 we have

$$
\varepsilon d\left[(x, y),\left(x^{*}, y^{*}\right)\right] \leq \varepsilon t \psi(\varepsilon)
$$

for every $\varepsilon \in[0,1]$. We can replace $\varepsilon$, for each $n \in \mathbb{N}$, by the sequence $[0,1] \ni \varepsilon_{n} \rightarrow 0$ as $n \rightarrow \infty$, which forces $d\left[(x, y),\left(x^{*}, y^{*}\right)\right]=0$.

Remark 3.4 Note that Theorem 2.2 is stronger than Theorem 2.1 in [16]. Indeed with the hypothesis of Theorem 2.1 in [16], for each $(x, y),(u, v) \in X \times X$, we have

$$
d(F(x, y), F(u, v)) \leq \frac{\lambda}{2} d[(x, y),(u, v)] \text { for all } x \geq u, y \leq v, 0 \leq \lambda<1 .
$$

Thus for $\alpha=\beta=1, \psi(\varepsilon)=\varepsilon$, and $\Lambda=\Lambda(\nu, \lambda)=\frac{v^{v}}{(1+\nu)^{1+v}(1-\lambda)^{v}}$, for arbitrary $v>0$, we get

$$
d(F(x, y), F(u, v)) \leq \frac{(1-\varepsilon)}{2} d[(x, y),(u, v)]+\Lambda \varepsilon^{1+\nu}[1+\|x, y\|+\|u, v\|],
$$

for every $\varepsilon \in[0,1]$.

\section{Competing interests}

The authors declare that they have no competing interests.

\section{Authors' contributions}

All authors contributed equally to the writing of this paper. All authors read and approved the final manuscript.

\section{Author details}

'Department of Mathematics, Faculty of Basic Sciences, Semnan University, P.O. Box 35195-363, Semnan, Iran. ${ }^{2}$ Young Researchers Club, Semnan Branch, Islamic Azad University, Semnan, Iran. ${ }^{3}$ Institute of Research and Development of Processes, University of Basque Country, Campus of Leioa (Bizkaia) - Aptdo. 644- Bilbao, Bilbao, 48080, Spain.

${ }^{4}$ Department of Mathematics, Kangnam University, Kangnam, Korea.

Received: 22 November 2013 Accepted: 8 May 2014 Published: 02 Jun 2014

\section{References}

1. Banach, S: Sur les opérations dans les ensembles abstraits et leur application aux équation intégrales. Fundam. Math. 3, 133-181 (1922)

2. Mizoguchi, N, Takahashi, W: Fixed point theorems for multivalued mappings on complete metric spaces. J. Math. Anal. Appl. 141, 177-188 (1989)

3. Nadler, SB: Multi-valued contraction mappings. Pac. J. Math. 30, 475-488 (1969)

4. Reich, S: Some problems and results in fixed point theory. Contemp. Math. 21, 179-187 (1983)

5. Samet, B, Vetro, C: Coupled fixed point theorems for multi-valued nonlinear contraction mappings in partially ordered metric spaces. Nonlinear Anal. 74(12), 4260-4268 (2011)

6. Samet, B, Karapinar, E, Aydi, H, Rajic, V: Discussion on some coupled fixed point theorems. Fixed Point Theory Appl. 2013, 50 (2013)

7. Gordji, ME, Ramezani, M: A generalization of Mizoguchi and Takahashi's theorem for single-valued mappings in partially ordered metric spaces. Nonlinear Anal. 74(13), 4544-4549 (2011)

8. Lakshmikantham, V, Ćirić, L: Coupled fixed point theorems for nonlinear contractions in partially ordered metric spaces. Nonlinear Anal. 70(12), 4341-4349 (2009) 
9. Radenović, S: Bhaskar-Lakshmikantham type results for monotone mappings in partially ordered metric spaces. Int. J. Nonlinear Anal. Appl. 5(2), 96-103 (2014)

10. Amini-Harandi, A, Emami, $\mathrm{H}$ : A fixed point theorem for contraction type maps in partially ordered metric spaces and application to ordinary differential equations. Nonlinear Anal. 72(5), 2238-2242 (2010)

11. Nieto, JJ, Rodriguez-Lopez, R: Contractive mapping theorems in partially ordered sets and applications to ordinary differential equations. Order 22, 223-239 (2005)

12. Nieto, JJ, Rodriguez-Lopez, R: Existence and uniqueness of fixed point in partially ordered sets and applications to ordinary differential equations. Acta Math. Sin. 23, 2205-2212 (2007)

13. Ran, ACM, Reurings, MCB: A fixed point theorem in partially ordered sets and some applications to matrix equations. Proc. Am. Math. Soc. 132, 1435-1443 (2004)

14. Pata, V: A fixed point theorem in metric spaces. J. Fixed Point Theory Appl. 10, 299-305 (2011)

15. Paknazar, M, Eshaghi, M, Cho, YJ, Vaezpour, SM: A Pata-type fixed point theorem in modular spaces with application. Fixed Point Theory Appl. 2013, 239 (2013). doi:10.1186/1687-1812-2013-239

16. Gnana Bhaskar, T, Lakshmikantham, V: Fixed point theorems in partially ordered metric spaces and applications. Nonlinear Anal. 65(7), 1379-1393 (2006)

10.1186/1687-1812-2014-130

Cite this article as: Eshaghi et al.: Pata contractions and coupled type fixed points. Fixed Point Theory and Applications 2014, 2014:130

\section{Submit your manuscript to a SpringerOpen ${ }^{\circ}$ journal and benefit from:}

- Convenient online submission

Rigorous peer review

- Immediate publication on acceptance

- Open access: articles freely available online

- High visibility within the field

- Retaining the copyright to your article 